\title{
Phosphorylated protein phosphatase 2A determines poor outcome in patients with metastatic colorectal cancer
}

\author{
I Cristóbal ${ }^{\star}, 1$, R Manso ${ }^{2}$, R Rincón ${ }^{1}, \mathrm{C}$ Caramés ${ }^{1}, \mathrm{~S}$ Zazo $^{2}$, T G del Pulgar ${ }^{1}$, A Cebrián ${ }^{1}$, J Madoz-Gúrpide ${ }^{2}$, \\ F Rojo ${ }^{\star, 2}$ and J García-Foncillas ${ }^{\star 1}$ \\ ${ }^{1}$ Translational Oncology Division, Oncohealth Institute, IIS-Fundacion Jimenez Diaz, UAM, University Hospital 'Fundacion Jimenez \\ Diaz', E-28040 Madrid, Spain and 'Pathology Department, University Hospital 'Fundacion Jimenez Diaz', Autonomous University \\ of Madrid, E-28040 Madrid, Spain
}

Background: Protein phosphatase 2A (PP2A) is a tumour suppressor frequently inactivated in human cancer and its tyrosine-307 phosphorylation has been reported as a molecular inhibitory mechanism.

Methods: Expression of phosphorylated PP2A (p-PP2A) was evaluated in 250 metastatic colorectal cancer (CRC) patients. Chi-square, Kaplan-Meier and Cox analyses were used to determine correlations with clinical and molecular parameters and impact on clinical outcomes.

Results: High p-PP2A levels were found in 17.2\% cases and were associated with ECOG performance status $(P=0.001)$ and presence of synchronous metastasis at diagnosis $(P=0.035)$. This subgroup showed substantially worse overall survival (OS) (median OS, 6.0 vs 26.2 months, $P<0.001$ ) and progression-free survival (PFS) (median PFS, 3.8 vs 13.3 months, P<0.001). The prognostic impact of $\mathrm{P}-\mathrm{PP} 2 \mathrm{~A}$ was particularly evident in patients aged $<70$ years $(P<0.001)$. Multivariate analysis revealed that P-PP2A retained its prognostic impact for OS (hazard ratio $2.7 ; 95 \%$ confidence interval, $1.8-4.1 ; P<0.001)$ and PFS (hazard ratio 3.0; $95 \%$ confidence interval, 1.8-5.0; $P<0.001)$.

Conclusions: Phosphorylated PP2A is an alteration that determines poor outcome in metastatic CRC and represents a novel potential therapeutic target in this disease, thus enabling to define a subgroup of patients who could benefit from future treatments based on PP2A activators.

Colorectal cancer (CRC) is the most common gastrointestinal cancer and its aetiology involves an interaction of genetic and epigenetic alterations with environmental factors such as diet components that contribute to cancer development. A correct balance between kinase and phosphatase activities is essential in maintaining cell homeostasis (Hunter, 1995). The presence of alterations affecting kinase activities has been shown to be recurrent in many cancers, and therapies based on kinase inhibitors have been developed in the previous years. Although the role of phosphatases remains underexplored in comparison, it is true that phosphatases like protein phosphatase $2 \mathrm{~A}$ (PP2A) have progressively been considered as potential tumour suppressors.
Protein phosphatase 2A is a major serine/threonine phosphatase that consists of a heterotrimer that includes a catalytic, a scaffold, and a regulatory subunit involved in the specificity and localisation of the holoenzyme. Owing to the multiple existing isoforms and splicing variants for each PP2A subunit, especially in the case of the regulatory subunit, PP2A can form a wide variety of heterotrimeric complexes with distinct substrate specificities and they therefore have different biological functions (Arino et al, 1988; Zhou et al, 2003; Eichhorn et al, 2009). Of importance, PP2A plays a pivotal role in regulating many signalling pathways (Millward et al, 1999; Janssens and Goris, 2001; Janssens et al, 2005; Mumby, 2007) and its inactivation has been described as a common event in

*Correspondence: Dr I Cristobal; E-mail: ion.cristobal@fjd.es or Dr F Rojo; E-mail: frojo@fjd.es or Professor J García-Foncillas; E-mail: jgfoncillas@fjd.es Received 27 January 2014; revised 20 May 2014; accepted 13 June 2014; published online 8 July 2014 
the cancerous cell through several molecular strategies (Mumby, 2007; Westermarck and Hahn, 2008; Eichhorn et al, 2009). Interestingly, Chen et al (1992) demonstrated that in vitro phosphorylation of PP2A at Y307 by protein tyrosine kinases led to its inactivation. Moreover, phosphorylation of PP2A at Y307 has been described as a molecular PP2A-inactivating mechanism with relevance in chronic and acute leukaemias or Alzheimer's disease (Perrotti and Neviani, 2008; Cristobal et al, 2010, 2011; Xiong et al, 2013). Although PP2A has been proposed as a novel therapeutic target in several tumours (Perrotti and Neviani, 2008, 2013; Kalev and Sablina, 2011; Voronkov et al, 2011), the potential relevance of PP2A as a druggable tumour suppressor in CRC still needs to be clarified. However, several observations indicate that PP2A inhibition could be playing an important role in CRC development. Thus, the presence of reported PP2A-inactivating mutations affecting the scaffold PP2A subunit in CRC (Wang et al, 1998; Takagi et al, 2000; Ruediger et al, 2001; Tamaki et al, 2004), together with the fact that PP2A seems to modulate the sensitivity of CRC cells to different treatments (Tan et al, 2010; Kumar et al, 2012; Lin et al, 2012), prompted us to hypothesise that PP2A could represent a novel molecular target with relevance in CRC.

In this report, we studied p-PP2A in a cohort of 250 metastatic CRC patients, observing that high p-PP2A levels were associated with worse ECOG performance status and the presence of synchronous metastasis. Of importance, the high p-PP2A subgroup showed a markedly shorter overall survival (OS) and progression-free survival (PFS), with significance in both wild-type and mutated KRAS subgroups. Interestingly, multivariate analysis showed that p-PP2A has an independent prognostic value for OS and PFS in metastatic CRC.

\section{MATERIALS AND METHODS}

Patient samples. The study comprised consecutive formalin-fixed paraffin-embedded tumour samples of 250 patients with metastatic CRC that were retrospectively selected from 2001 to 2012 according to the following criteria: adenocarcinoma, operable disease, no neoadjuvant therapy, enough available tissue, clinical follow-up data and metastatic disease. Samples were surgical resection specimens from CRC tumours obtained from Fundacion Jimenez Diaz Biobank (BFJD, Madrid, Spain). TNM (tumour, Node, Metastases) staging was classified using the 7th American Joint Committee on Cancer staging system for CRC. Clinical data were collected from medical clinical records by oncologists (JG-F, CC). KRAS mutational status was determined using the Cobas KRAS Mutation Test kit (Roche Molecular Diagnostics, Branchburg, NJ, USA) following manufacturer's procedures. Tissue microarrays (TMAs) were constructed. Representative areas of each tumour were carefully selected and three tissue cores ( $1 \mathrm{~mm}$ diameter) were obtained using a TMA workstation (T1000 Chemicon, Temecula, CA, USA). Samples were taken anonymously. The ethics committee and institutional review board approved the project.

Immunohistochemistry. Tissue sections $(3 \mu \mathrm{m})$ were placed on plus-charged glass slides. After deparaffinisation in xylene and graded alcohols, heat antigen retrieval was performed in $\mathrm{pH} 9$ EDTA-based buffer (Dako, Glostrup, Denmark). Endogenous peroxidase was blocked by using $0.03 \%$ hydrogen peroxide for $5 \mathrm{~min}$. Slides were incubated with the primary antibody against p-PP2A (rabbit monoclonal anti-p-PP2A Y307; Abcam, Cambridge, UK; ref.: ab32104) for $60 \mathrm{~min}$ at room temperature, followed by the appropriate anti-Ig horseradish peroxidaseconjugated polymer (Flex + , Dako). Sections were visualised with 3,3'-diaminobenzidine as a chromogen. All stainings were performed in a Dako Autostainer. Sections incubated with normal non-immunised rabbit immunoglobulin were used as negative controls. As positive control, a section of colorectal tumour with known expression of p-PP2A was used. p-PP2A antibody sensitivity $(1: 2000)$ had been calculated in a range of crescent dilutions of the primary antibody. Specificity was confirmed in a set of paired fresh frozen and formalin-fixed paraffin-embedded samples processed by western blot and IHC (Supplementary Figure 1). Moreover, additional controls to confirm the specificity of the antibody were performed and included PP2A silencing with specific siRNAs designed and synthesised by Dharmacon RNA Technologies (Dharmacon, Lafayette, CO, USA), in vitro dephosphorylation of SW480 and HT-29 cell lysates using calf intestinal phosphatase (CIP), and western blot analysis of CRC tumour tissues showing the entire membrane to demonstrate that any other major bands are recognised by the antibody (Supplementary Figure 2). Antibodies used were mouse monoclonal anti-PP2A (Upstate Inc., Lake Placid, NY, USA) and mouse monoclonal anti$\beta$ actin (Sigma, St Louis, MO, USA). Antigen preservation in tissues was confirmed by assaying sections from the same TMAs for expression of phospho-tyrosines by immunohistochemistry, using a monoclonal antibody to tyrosine-phosphorylated proteins (clone 4G10, 1:500, Millipore, Billerica, MA, USA). Those cases showing absent, irregular, or weak staining for the phospho-tyrosine assay were excluded from the analysis. Only the membrane of epithelial cells, but not stromal cells, was evaluated for $\mathrm{p}-\mathrm{PP} 2 \mathrm{~A}$ expression in a manner blinded to clinical data by two pathologists (FR and SZ). A semiquantitative histoscore was calculated by estimating the percentage of tumour cells positively stained with low, medium, or high staining intensity. The final score was determined after applying a weighting factor to each estimate. The following formula was used: histoscore $=($ low $\%) \times 1+($ medium $\%) \times 2+$ $($ high $\%) \times 3$ and the results ranged from 0 to 300 .

Statistical analysis. Statistical analyses were performed using SPSS 20 for windows (SPSS Inc, Chicago, IL, USA). Overall survival was defined as the time from the date of metastatic diagnosis to the date of death from any cause or last follow-up. Progression-free survival was defined as the time from metastatic diagnosis until progression to first-line metastatic treatment or death. The Kaplan-Meier method and survival comparisons were done with the log-rank test if proportional hazard assumption was fulfilled and Breslow otherwise. The Cox proportional hazards model was adjusted by taking into consideration significant parameters in univariate analysis. A $P$-value $<0.05$ was considered statistically significant. Receiver operating curve was used to determine the optimal cutoff point based on progression end point for $\mathrm{p}-\mathrm{PP} 2 \mathrm{~A}$ expression as previously described (Generali et al, 2009). This work was carried out in accordance with the Reporting Recommendations for Tumor Marker Prognostic Studies guidelines (McShane et al, 2005).

\section{RESULTS}

Prevalence of p-PP2A in metastatic CRC and its association with clinical and molecular parameters. To study the prevalence of $\mathrm{p}-\mathrm{PP} 2 \mathrm{~A}$ and its potential clinical significance in CRC, we quantified the expression of p-PP2A by immunohistochemistry in a series of 250 patients with metastatic CRC, correlated the results obtained with clinical and molecular features, and studied the prognostic relevance of this aberration. Patient characteristics are presented in Supplementary Table 1. High p-PP2A expression was observed in $17.2 \%$ cases (43 out of 250 ). The prevalence of PP2A hyperphosphorylation was higher in women than in men (24.5\% vs $12.8 \%, P=0.018)$. Moreover, we found high $\mathrm{p}-\mathrm{PP} 2 \mathrm{~A}$ to be associated with worse ECOG performance status $(35.7 \%$ vs $15.5 \%, P=0.001)$, and with the presence of synchronous metastasis $(20.9 \%$ vs $11.3 \%, P=0.035)$. Association between p-PP2A and clinical and genetic parameters is shown in Table 1. 
Table 1. Association between P-PP2A and clinical and genetic parameters in 250 patients with metastatic CRC

\begin{tabular}{|c|c|c|c|c|}
\hline & No. of cases & No. p-PP2A - (\%) & No. p-PP2A + (\%) & $P$ \\
\hline p-PP2A & 250 & $207(82.8)$ & $43(17.2)$ & \\
\hline Sex & 250 & 207 & 43 & 0.018 \\
\hline Male & 156 & $136(87.2)$ & $20(12.8)$ & \\
\hline Female & 94 & $71(75.5)$ & $23(24.5)$ & \\
\hline Age & 232 & 190 & 42 & 0.306 \\
\hline$<70$ & 116 & $98(84.5)$ & $18(15.5)$ & \\
\hline$\geqslant 70$ & 116 & $92(79.3)$ & $24(20.7)$ & \\
\hline ECOG & 224 & 184 & 40 & 0.001 \\
\hline $0-2$ & 182 & $157(84.5)$ & 25 (15.5) & \\
\hline $3-4$ & 42 & 27 (64.3) & $15(35.7)$ & \\
\hline Site of primary tumour & 250 & 207 & 43 & 0.552 \\
\hline Cecum & 24 & $17(84.5)$ & $7(15.5)$ & \\
\hline Right colon & 37 & $31(79.3)$ & $6(20.7)$ & \\
\hline Transverse colon & 10 & $7(70)$ & $3(30)$ & \\
\hline Left colon & 21 & $18(85.7)$ & $3(14.3)$ & \\
\hline Sigma & 73 & $61(83.6)$ & $12(16.4)$ & \\
\hline Rectum & 85 & $73(85.9)$ & $12(14.1)$ & \\
\hline Synchronous metastasis & 244 & 201 & 43 & 0.035 \\
\hline No & 85 & $76(89.4)$ & $9(10.6)$ & \\
\hline Yes & 159 & $125(78.6)$ & $34(21.4)$ & \\
\hline Number of metastatic sites & 250 & 207 & 43 & 0.620 \\
\hline $1-2$ & 226 & $188(83.2)$ & $38(16.8)$ & \\
\hline$>2$ & 24 & 19 (79.2) & $5(20.8)$ & \\
\hline Liver metastasis & 244 & 201 & 43 & 0.294 \\
\hline No & 79 & $68(86.1)$ & $11(13.9)$ & \\
\hline Yes & 165 & $133(80.6)$ & $32(19.4)$ & \\
\hline Lung metastasis & 244 & 201 & 43 & 0.323 \\
\hline No & 166 & $134(80.7)$ & $32(19.3)$ & \\
\hline Yes & 78 & $67(85.9)$ & $11(14.1)$ & \\
\hline Lymph metastasis & 244 & 201 & 43 & 0.409 \\
\hline No & 177 & $148(83.6)$ & $29(16.4)$ & \\
\hline Yes & 67 & $53(79.1)$ & $14(20.9)$ & \\
\hline Peritoneal metastasis & 244 & 201 & 43 & 0.846 \\
\hline No & 196 & $161(82.1)$ & $35(17.9)$ & \\
\hline Yes & 48 & 40 (83.3) & $8(16.7)$ & \\
\hline MSI & 240 & 189 & 41 & 0.774 \\
\hline No & 226 & $187(82.7)$ & $39(17.3)$ & \\
\hline Yes & 14 & $12(85.7)$ & $2(14.3)$ & \\
\hline KRAS mutations & 246 & 203 & 43 & 0.873 \\
\hline No & 140 & $116(82.9)$ & $24(17.1)$ & \\
\hline Yes & 106 & 87 (72.1) & 19 (17.9) & \\
\hline
\end{tabular}

Clinical significance of p-PP2A in metastatic CRC. Clinical follow-up data were available for 243 cases, 149 men and 94 women, with a median age of 69.5 years (age range: 29-92). Median OS of the global cohort was 21.9 months (95\% confidence interval, 17.2-26.6 months). We found that the subgroup of patients with high $\mathrm{p}-\mathrm{PP} 2 \mathrm{~A}$ showed a substantially shorter OS (median OS, 6.0 vs 26.2 months, $P<0.001$ ) (Figure 1A) and PFS (median PFS, 3.8 vs 13.3 months, $P<0.001$ ) (Figure 1B). Interestingly, the prognostic impact of p-PP2A was particularly evident in the subgroup of patients aged $<70$ years (median OS, 6.2 vs 33.2 months, $P<0.001$; median PFS, 4.4 vs 16.4 months, $P<0.001$ ); however, significance was also achieved in the subgroup of elderly patients (median OS, 5.9 vs 15.2 months, $P=0.012$; median PFS, 3.8 vs 7.6 months, $P=0.020$ ) (Figure 2 ). To further investigate the 

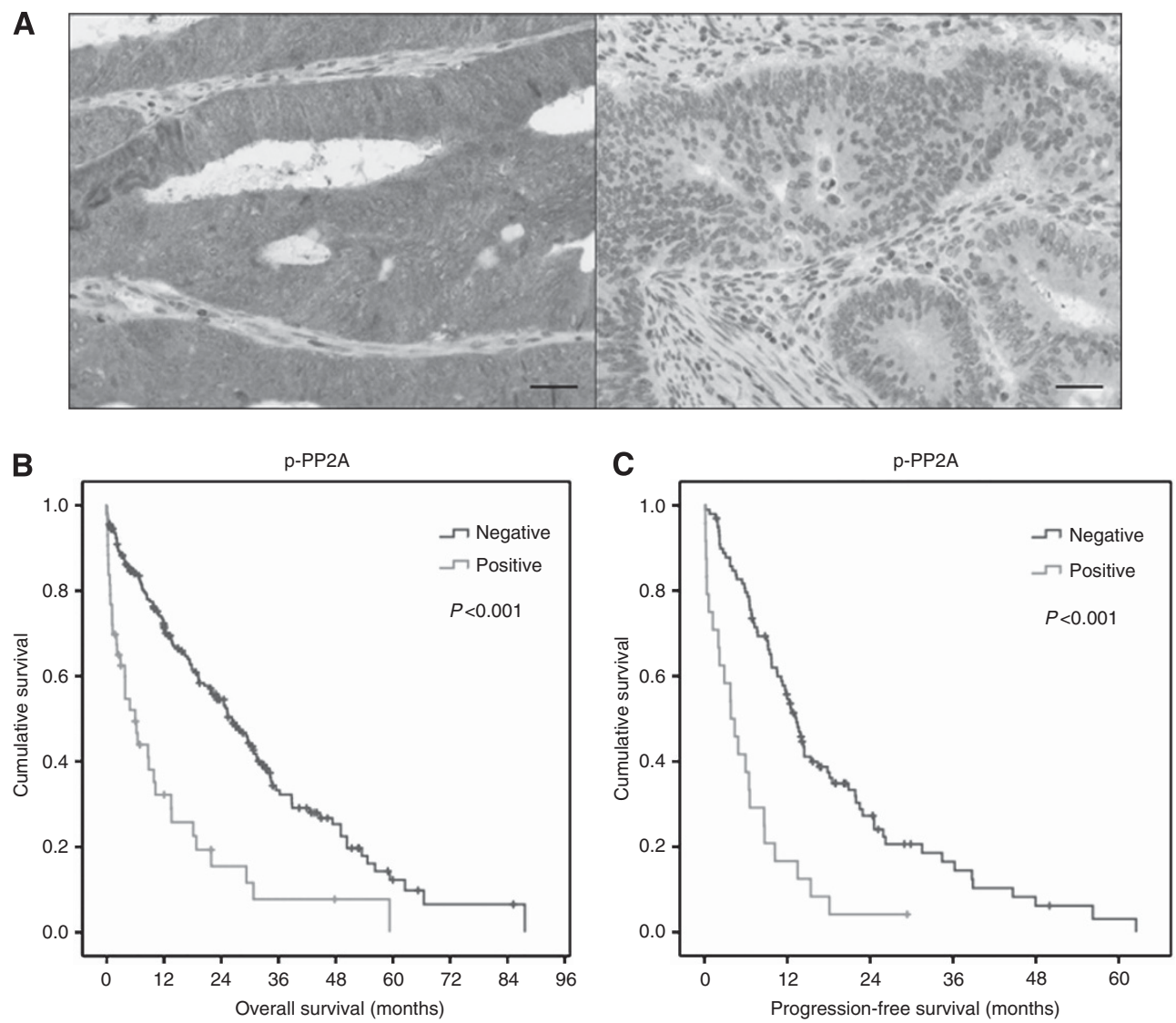

Figure 1. Clinical significance of p-PP2A in metastatic CRC: (A) Immunohistochemical detection of p-PP2A showing positive and negative staining. The line shows $25 \mu \mathrm{m}$. Magnification $\times 400$; Kaplan-Meier analyses of overall survival (B) and progression-free survival (C) in a cohort of 243 patients with metastatic CRC.
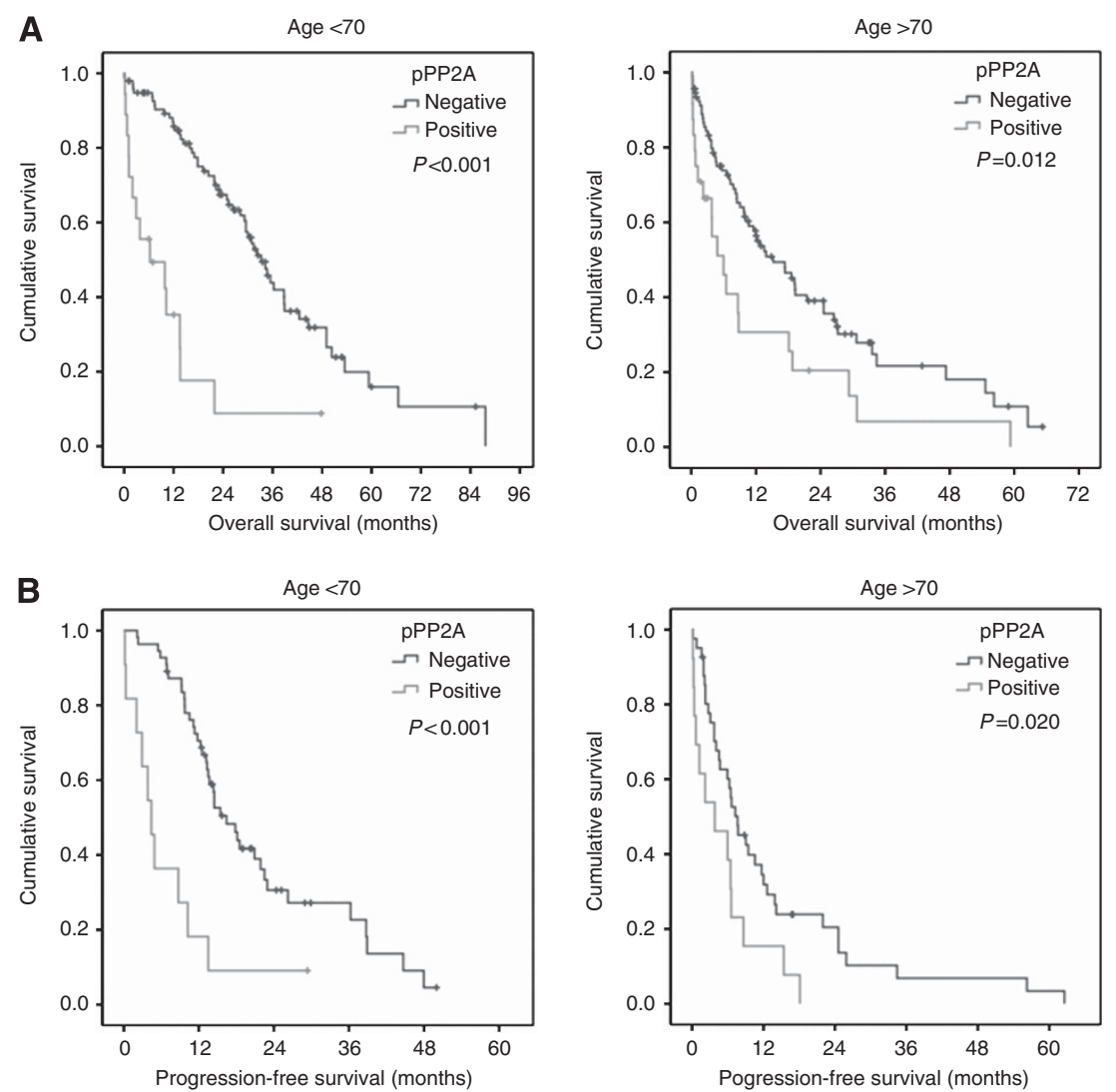

Figure 2. Kaplan-Meier analyses in the subgroups of patients aged $<$ and $>70$ years: (A) Overall survival; (B) progression-free survival. 


\begin{tabular}{|c|c|c|c|c|c|c|}
\hline & \multicolumn{3}{|c|}{ Univariate OS analysis } & \multicolumn{3}{|c|}{ Multivariate OS Cox analysis } \\
\hline & HR & $\begin{array}{c}95 \% \mathrm{Cl} \\
\text { Lower-upper }\end{array}$ & Significance & $H R$ & $\begin{array}{c}95 \% \mathrm{Cl} \\
\text { Lower-upper }\end{array}$ & Significance \\
\hline Age & & & $<0.001$ & & & 0.249 \\
\hline & 1.000 & & & 1.000 & & \\
\hline & 1.875 & $1.352-2.599$ & & 1.250 & $0.855-1.827$ & \\
\hline Gender & & & 0.311 & & - & \\
\hline Male & 1.000 & & & & & \\
\hline Female & 0.848 & $0.616-1.167$ & & - & - & \\
\hline Synchronous & & & 0.118 & & - & \\
\hline No & 1.000 & & & & & \\
\hline Yes & 1.301 & $0.935-1.811$ & & - & - & \\
\hline ECOG & & & $<0.001$ & & & $<0.001$ \\
\hline $0-1$ & 1.000 & & & 1.000 & & \\
\hline $2-3$ & 1.925 & $1.588-2.333$ & & 1.777 & $1.427-2.213$ & \\
\hline MSI & & & 0.450 & & - & \\
\hline No & 1.000 & & & & & \\
\hline Yes & 1.281 & $0.674-2.437$ & & - & - & \\
\hline Number of metastatic sites & & & 0.076 & & - & \\
\hline $1-2$ & 1.000 & & & & & \\
\hline$>2$ & 1.250 & $0.977-1.600$ & & - & - & \\
\hline p-PP2A & & & $<0.001$ & & & $<0.001$ \\
\hline No & 1.000 & & & 1.000 & & \\
\hline Yes & 2.838 & $1.935-4.164$ & & 2.743 & 1.819-4.138 & \\
\hline
\end{tabular}

clinical relevance of $\mathrm{p}-\mathrm{PP} 2 \mathrm{~A}$ in metastatic $\mathrm{CRC}$ we analysed its potential prognostic value by stratifying our cohort based on KRAS mutation status, and we found that $\mathrm{p}$-PP2A retained its prognostic impact in both KRAS wild-type and KRAS mutated subgroups with a similar significance (Supplementary Figure 3). Importantly, multivariate analysis demonstrated that P-PP2A is an unfavourable independent factor associated with OS (hazard ratio 2.7; 95\% confidence interval, 1.8-4.1; $P<0.001$ ) (Table 2) and PFS (hazard ratio 3.0 ; $95 \%$ confidence interval, $1.8-5.0 ; P<0.001$ ) (Table 3 ) in metastatic CRC.

\section{DISCUSSION}

We report here that $\mathrm{PP} 2 \mathrm{~A}$ hyperphosphorylation is a recurrent molecular event in metastatic CRC associated with worse ECOG performance status and the presence of synchronous metastasis. Importantly, this alteration determines a markedly shorter overall and PFS, especially in the subgroup of patients younger than 70 years. The prognostic impact was similar in the KRAS wild-type and mutated subgroups. Moreover, multivariate analysis showed that high p-PP2A expression has an independent prognostic value for OS and PFS in patients with metastatic CRC. Of importance, our data provide strong evidence that $\mathrm{p}-\mathrm{PP} 2 \mathrm{~A}$ has a potential prognostic value and could be a promising therapeutic target for future clinical trials using PP2A activators.

Despite progressive advances in our understanding of the molecular biology of CRC, patient outcomes in the metastatic subgroup are still very poor. Therefore, it is necessary to develop alternative therapeutic strategies to improve the survival of these patients. The tumour suppressor PP2A has been shown to be functionally inactivated in several types of human cancer through different contributing mechanisms, including the hyperphosphorylation of its catalytic subunit (Saydam et al, 2003; Cristobal et al, 2011). However, in comparison with other tumour models, the relevance of the tumour suppressor role of PP2A and its potential clinical significance in CRC remains mostly unknown. Therefore, to evaluate the clinical relevance of PP2A phosphorylation in metastatic CRC, we analysed the expression of p-PP2A in a cohort of 250 patients with metastatic CRC, observing high p-PP2A in $17.2 \%$ of cases (Table 1). The prevalence observed for this alteration in our cohort suggests that this would be a relevant molecular mechanism to inactivate PP2A in CRC.

Moreover, we observed that high p-PP2A correlated positively with a high grade of ECOG performance status and with the existence of synchronous metastasis at diagnosis in our cohort (Table 1). These results prompted us to hypothesise that this could be a molecular alteration characteristic of the advanced stages of CRC that could therefore have a prognostic value in patients with metastatic disease. In concordance with this, we observed that the subgroup of patients with high p-PP2A showed a substantially shorter OS and PFS compared with the low-p-PP2A subgroup (Figure 1), confirming the clinical relevance of $\mathrm{p}-\mathrm{PP} 2 \mathrm{~A}$ in metastatic CRC. Moreover, the fact that the prognostic impact of p-PP2A showed higher significance in the subgroup of patients aged $<70$ years (Figure 2) is very interesting since this subgroup includes cases with more options from a therapeutic perspective that could benefit from the treatment with PP2A activating drugs 


\begin{tabular}{|c|c|c|c|c|c|c|}
\hline & \multicolumn{3}{|c|}{ Univariate PFS analysis } & \multicolumn{3}{|c|}{ Multivariate PFS Cox analysis } \\
\hline & |HR & $\begin{array}{c}95 \% \mathrm{Cl} \\
\text { Lower-upper }\end{array}$ & Significance & HR & $\begin{array}{c}95 \% \mathrm{Cl} \\
\text { Lower-upper }\end{array}$ & Significance \\
\hline Age & & & 0.001 & & & 0.096 \\
\hline & 1.000 & & & 1.000 & & \\
\hline & 1.966 & $1.318-2.932$ & & 1.476 & $0.933-2.333$ & \\
\hline Gender & & & 0.595 & & - & \\
\hline Male & 1.000 & & & & & \\
\hline Female & 0.897 & $0.601-1.339$ & & - & - & \\
\hline Synchronous & & & 0.056 & & - & \\
\hline No & 1.000 & & & & & \\
\hline Yes & 1.522 & $0.990-2.341$ & & - & - & \\
\hline ECOG & & & $<0.001$ & & & 0.012 \\
\hline $0-1$ & 1.000 & & & 1.000 & & \\
\hline $2-3$ & 1.556 & $1.224-1.978$ & & 1.420 & $1.081-1.866$ & \\
\hline MSI & & & 0.426 & & - & \\
\hline No & 1.000 & & & & & \\
\hline Yes & 1.445 & $0.584-3.577$ & & - & - & \\
\hline Number of metastatic sites & & & 0.029 & & & 0.003 \\
\hline $1-2$ & 1.000 & & & 1.000 & & \\
\hline$>2$ & 1.374 & $1.033-1.827$ & & 1.564 & $1.165-2.099$ & \\
\hline p-PP2A & & & $<0.001$ & & & $<0.001$ \\
\hline No & 1.000 & & & 1.000 & & \\
\hline Yes & 3.046 & $1.885-4.822$ & & 3.008 & $1.804-5.016$ & \\
\hline
\end{tabular}

(e.g. FTY720), which have shown promising effects in other cancers (Kalev and Sablina, 2011; Perrotti and Neviani, 2013).

As the mutation status of KRAS has a strong predictive value of response to cetuximab in CRC, we stratified our series by KRAS mutation status and observed that $\mathrm{p}-\mathrm{PP} 2 \mathrm{~A}$ had a similar prognostic impact in both KRAS wild-type and KRAS mutated subgroups (Supplementary Figure 3). This result would indicate that the treatment with PP2A activators could also be a therapeutic option for those patients with high $\mathrm{p}-\mathrm{PP} 2 \mathrm{~A}$ in whom cetuximab is not recommended or in those cases with cetuximab resistance. Interestingly, it has recently been reported that FTY720 could resensitise CRC cells to cetuximab, indicating a potential therapeutic relevance for FTY720 in metastatic CRC (Rosa et al, 2013). Our group has recently reported overexpression of the PP2A inhibitors SET and $\mathrm{CIP} 2 \mathrm{~A}$ and downregulation of $\mathrm{PP} 2 \mathrm{~A}$ regulatory subunits such as PPP2R2A and PPP2R5E as the contributing mechanisms to PP2A inhibition in CRC (Cristóbal et al, 2014); some of these alterations could be present in the low-p-PP2A subgroup of CRC patients. Therefore, additional investigations are needed to clarify the presence and potential clinical significance of these other PP2A-inactivating mechanisms in the subgroup of CRC patients with low p-PP2A levels. Furthermore, it has been reported that PP2A downregulation is a relevant event in some human cancers such as acute myeloid leukaemia and prostate cancer (Cristobal et al, 2011; Bhardwaj et al, 2014). However, our previous investigations did not show PP2A downregulated in CRC, suggesting that this alteration, if present, would be of low prevalence in CRC. Anyway, it would be of interest to further analyzed in future studies whether this alteration could be present in a subgroup of CRC patients.
In conclusion, we show that $\mathrm{p}-\mathrm{PP} 2 \mathrm{~A}$ is a common alteration with clinical significance in metastatic CRC. Of importance, p-PP2A could serve as a novel molecular target that can help define a subgroup of metastatic CRC patients with worse outcome that could benefit by the future incorporation of PP2A-activating drugs in anticancer protocols.

\section{ACKNOWLEDGEMENTS}

This study was funded by the Biobank of Fundación Jiménez Díaz (FJD Biobank) (RD12/0036/0021), and PI12/01552 and PI13/ 02609 grants from Instituto de Salud Carlos III FEDER and S2010/ BMD2344. R Manso is supported by the Fundación Conchita Rábago de Jiménez Díaz.

\section{CONFLICT OF INTEREST}

The authors declare no conflict of interest.

\section{REFERENCES}

Arino J, Woon CW, Brautigan DL, Miller Jr TB, Johnson GL (1988) Human liver phosphatase 2A: cDNA and amino acid sequence of two catalytic subunit isotypes. Proc Natl Acad Sci USA 85(12): 4252-4256.

Bhardwaj A, Singh S, Srivastava SK, Arora S, Hyde SJ, Andrews J, Grizzle WE, Singh AP (2014) Restoration of PPP2CA expression 
reverses epithelial-to-mesenchymal transition and suppresses prostate tumour growth and metastasis in an orthotopic mouse model. Br J Cancer 110(8): 2000-2010.

Chen J, Martin BL, Brautigan DL (1992) Regulation of protein serinethreonine phosphatase type-2A by tyrosine phosphorylation. Science 257(5074): 1261-1264.

Cristobal I, Blanco FJ, Garcia-Orti L, Marcotegui N, Vicente C, Rifon J, Novo FJ, Bandres E, Calasanz MJ, Bernabeu C, Odero MD (2010) SETBP1 overexpression is a novel leukemogenic mechanism that predicts adverse outcome in elderly patients with acute myeloid leukemia. Blood 115(3): 615-625.

Cristobal I, Garcia-Orti L, Cirauqui C, Alonso MM, Calasanz MJ, Odero MD (2011) PP2A impaired activity is a common event in acute myeloid leukemia and its activation by forskolin has a potent anti-leukemic effect. Leukemia 25(4): 606-614.

Cristóbal I, Manso R, Rincón R, Caramés C, Senin C, Borrero A, Martínez-Useros J, Rodriguez M, Zazo S, Aguilera O, Madoz-Gúrpide J, Rojo F, García-Foncillas J (2014) PP2A inhibition is a common event in colorectal cancer and its restoration using FTY720 shows promising therapeutic potential. Mol Cancer Ther 13(4): 938-947.

Eichhorn PJ, Creyghton MP, Bernards R (2009) Protein phosphatase 2A regulatory subunits and cancer. Biochim Biophys Acta 1795(1): 1-15.

Generali D, Buffa FM, Berruti A, Brizzi MP, Campo L, Bonardi S, Bersiga A, Allevi G, Milani M, Aguggini S, Papotti M, Dogliotti L, Bottini A, Harris AL, Fox SB (2009) Phosphorylated ERalpha, HIF-1alpha, and MAPK signaling as predictors of primary endocrine treatment response and 470 resistance in patients with breast cancer. J Clin Oncol 27(2): $227-234$.

Hunter T (1995) Protein kinases and phosphatases: the yin and yang of protein phosphorylation and signalling. Cell 80(2): 225-236.

Janssens V, Goris J (2001) Protein phosphatase 2A: a highly regulated family of serine/threonine phosphatases implicated in cell growth and signalling. Biochem J 353(Pt3): 417-439.

Janssens V, Goris J, Van Hoof C (2005) PP2A: the expected tumor suppressor. Curr Opin Genet Dev 15(1): 34-41.

Kalev P, Sablina AA (2011) Protein phosphatase 2A as a potential target for anticancer therapy. Anticancer Agents Med Chem 11(1): 38-46.

Kumar A, Pandurangan AK, Lu F, Fyrst H, Zhang M, Byun HS, Bittman R, Saba JD (2012) Chemopreventive sphingadienes downregulate Wnt signaling via a PP2A/Akt/GSK3 $\beta$ pathway in colon cancer. Carcinogenesis 33(9): 1726-1735.

Lin SP, Lee YT, Yang SH, Miller SA, Chiou SH, Hung MC, Hung SC (2012) Colon cancer stem cells resist antiangiogenesis therapy-induced apoptosis. Cancer Lett 328(2): 226-234.

McShane LM, Altman DG, Sauerbrei W, Taube SE, Gion M, Clark GM. Statistics Subcommittee of the NCI-EORTC Working Group on Cancer Diagnostics (2005) Reporting recommendations for tumor marker prognostic studies. J Clin Oncol 23(36): 9067-9072.

Millward TA, Zolnierowicz S, Hemmings BA (1999) Regulation of protein kinase cascades by protein phosphatase 2A. Trends Biochem Sci 24(5): 186-191.
Mumby M (2007) PP2A: unveiling a reluctant tumor suppressor. Cell 130(1): 21-24.

Perrotti D, Neviani P (2008) Protein phosphatase 2A (PP2A), a drugable tumor suppressor in $\mathrm{Ph} 1(+)$ leukemias. Cancer Metastasis Rev 27(2): $159-168$.

Perrotti D, Neviani P (2013) Protein phosphatise 2A: a target for anticancer therapy. Lancet Oncol 14(6): e229-e238.

Rosa R, Marciano R, Malapelle U, Formisano L, Nappi L, D'Amato C, D’Amato V, Damiano V, Marfè G, Del Vecchio S, Zannetti A, Greco A, De Stefano A, Carlomagno C, Veneziani BM, Troncone G, De Placido S, Bianco R (2013) Sphingosine kinase 1 overexpression contributes to cetuximab resistance in human colorectal cancer models. Clin Cancer Res 19(1): 138-147.

Ruediger R, Pham HT, Walter G (2001) Alterations in protein phosphatase 2A subunit interaction in human carcinomas of the lung and colon with mutations in the A beta subunit gene. Oncogene 20(15): 1892-1899.

Saydam G, Aydin HH, Sahin F, Selvi N, Oktem G, Terzioglu E, Buyukkececi F, Omay SB (2003) Involvement of protein phosphatase 2A in interferonalpha-2b-induced apoptosis in K562 human chronic myelogenous leukaemia cells. Leuk Res 27(8): 709-717.

Takagi Y, Futamura M, Yamaguchi K, Aoki S, Takahashi T, Saji S (2000) Alterations of the PPP2R1B gene located at 11q23 in human colorectal cancers. Gut 47(2): 268-271.

Tamaki M, Goi T, Hirono Y, Katayama K, Yamaguchi A (2004) PPP2R1B gene alterations inhibit interaction of PP2A-Abeta and PP2A-C proteins in colorectal cancers. Oncol Rep 11(3): 655-659.

Tan J, Lee PL, Li Z, Jiang X, Lim YC, Hooi SC, Yu Q (2010) B55 $\beta$-associated PP2A complex controls PDK1-directed myc signaling and modulates rapamycin sensitivity in colorectal cancer. Cancer Cell 18(5): 459-471.

Voronkov M, Braithwaite SP, Stock JB (2011) Phosphoprotein phosphatase 2A: a novel druggable target for Alzheimer's disease. Future Med Chem 3(7): 821-833.

Wang SS, Esplin ED, Li JL, Huang L, Gazdar A, Minna J, Evans GA (1998) Alterations of the PPP2R1B gene in human lung and colon cancer. Science 282(5387): 284-287.

Westermarck J, Hahn WC (2008) Multiple pathways regulated by the tumor suppressor PP2A in transformation. Trends Mol Med 14(4): 152-160.

Xiong Y, Jing XP, Zhou XW, Wang XL, Yang Y, Sun XY, Qiu M, Cao FY, Lu YM, Liu R, Wang JZ (2013) Zinc induces protein phosphatase 2A inactivation and tau hyperphosphorylation through Src dependent PP2A (tyrosine 307) phosphorylation. Neurobiol Aging 34(3): 745-756.

Zhou J, Pham HT, Ruediger R, Walter G (2003) Characterization of the Aalpha and Abeta subunit isoforms of protein phosphatase 2A: differences in expression, subunit interaction, and evolution. Biochem J 369(Pt2): 387-398.

This work is published under the standard license to publish agreement. After 12 months the work will become freely available and the license terms will switch to a Creative Commons AttributionNonCommercial-Share Alike 3.0 Unported License.

Supplementary Information accompanies this paper on British Journal of Cancer website (http://www.nature.com/bjc) 